

\section{La historia del rock en Colombia desde los años 60 hasta la actualidad (parte 1)}

\section{Artículo de reflexión}

Recibido: 15 de agosto de 2018

Aprobado: 20 de septiembre de 2018

\author{
Camilo Andrés Ortegón Munevar \\ Universidad Distrital Francisco José de Caldas, \\ Colombia \\ caom428@gmail.com
}

Cómo citar este artículo: Ortegón Munevar, Camilo Andrés (2019). La historia del rock en Colombia desde los años 60 hasta la actualidad (parte 1). Estudios Artísticos: revista de investigación creadora, 5(6) pp. 102-116. DOI: https://doi. org/10.14483/25009311.14106

\section{(c) (1)}

https://creativecommons.org/licenses/by/4.0/deed.es

$<$

Camilo Ortegón (2018). Práctica. Fotografía: Pedro Pablo Gómez.

\section{Resumen}

Este artículo se ocupa de la primera parte de una breve historia del rock en Colombia en el contexto más amplio del rock en Latinoamérica a partir de los años 60. Su propósito fundamental es visibilizar el trabajo de algunos bateristas que tuvieron un papel clave en la historia local de rock. Se trata de un texto realizado por un baterista interesado en el trabajo pionero de bateristas colombianos que aportaron interesantes ideas compositivas para el desarrollo del rock en Colombia.

\section{Palabras clave}

Batería; aportes creativos e interpretativos; rock colombiano; bateristas colombianos

\section{The History of Rock in Colombia from the 60s to the Present Day (Part 1)}

\begin{abstract}
This article deals with the first part of a brief history of rock in Colombia in the broader context Latin American Rock from the 60s onward. Its main purpose is to make visible the work of some drummers who played a key role in the history of local rock. What follows was penned by a drummer interested in the pioneering work of Colombian drummers who put forward interesting musical ideas for the development of rock in Colombia.
\end{abstract}

\section{Keywords}

Drums; creative and interpretative contributions; colombian rock; colombian drummers 


\section{L'histoire du rock en Colombie des années 60 à nos jours (partie 1)}

\section{Résumé}

Cet article contient la première partie d'une brève histoire du rock en Colombie dans le contexte plus large du rock en Amérique latine depuis les années 60 . Son objectif fondamental est de rendre visible le travail de certains batteurs qui ont joué un rôle clé dans I'histoire du rock local. Ce texte a été rédigé par un batteur intéressé par le travail pionnier de batteurs colombiens qui ont apporté d'intéressantes idées musicales pour le développement du rock en Colombie.

\section{Mots clés}

Batterie ; contributions créatives et interprétatives ; rock colombien; batteurs colombiens

\section{A história do rock na Colômbia dos anos 60 até o presente (parte 1)}

\section{Resumo}

Este artigo contém a primeira parte de uma breve história do rock na Colômbia no contexto mais amplo do rock na América Latina a partir dos anos 60. Seu objetivo fundamental é tornar visível o trabalho de alguns bateristas que desempenharam um papel fundamental na história do rock local. Este texto foi feito por um baterista interessado no trabalho pioneiro de bateristas colombianos que contribuíram com idéias interessantes de composição para o desenvolvimento do rock na Colômbia.

\section{Palavras-chaves}

Bateria; contribuições criativas e interpretativas; rock colombiano; bateristas colombianos

\section{Parlu kai virsai rock Kukumbia suti alpamanda kaska kai sugta chunga wata i kai puncha mana (su kaiarii)}

\section{Maillallachiska}

Kai parlukuna kaiarimi chi parlu kai sug visria rock suti kaskauramanda kai Kulumbia kai latinuamerikami ña kai sugta wata kunaura. Kai tsabaju kaiarimi kangapa imasami kai runakuna kaiariskakuna bunbua virsiai kai Kulumbiapi imasami paikunapas aidachirkakuna,rurangapa chi
Virsiangapa bombuan chi tsabajukuna kai Kulumbia suma alpapi.

\section{Rimangapa Ministidukuna}

Aparii; aidachinakui i iuichinakui; Kulumbiamanda

visiadurkuna ruckuwa; bumbua visrsiag

Klulumbiakuna

\section{Una mirada hacia Latinoamérica e Iberoamérica (México, Argentina, España, Brasil)}

Manolo Bellón, en su libro "El ABC del rock" expone que, por la década de 1930, en algunos círculos sociales de Estados Unidos, aunque los blancos y negros todavía sufrían una fuerte separación racial, ya se estaba empezando a crear un intercambio de música entre estos grupos. Y que para 1934, un grupo de investigadores del folclor norteamericano, padre e hijo de apellido Lomax, escuchan en una iglesia un ritmo constante y pulsante, con un canto melódico basado en los blues que entonaban los negros, con letras llenas de mensaje espiritual y conciencia social. Este evento cautivó a los investigadores, sin saber que 20 años más tarde, todo esto gestaría el Rock and Roll (Bellón, 2007, p. 31).

Años más tarde, ya cuando el Rock and Roll se había vuelto una fuerza imparable, por allá cerca de los años 60, logra llegar a territorio lberoamericano por medio de las cercanías con los países anglosajones. El primer indicio que conocemos del Rock en Iberoamérica fue una grabación hecha por Ritchie Valens de la canción "La bamba" en octubre del año 1958, la cual es una canción tradicional mexicana que tomaría un carácter de Rock and Roll. Esta canción fue un éxito durante los años 1958 y 1959, ocupando el puesto número de los Bilboard Top 100. Pero infortunadamente, la fuerza del Rock and Roll, fue disminuida y detenida en estos países, puesto que el pensamiento (en muchos casos errado) de que este género esta implícitamente atado al uso de drogas, hizo que menguara y se apagara su luz. De hecho, el estilo de la música rock que se hacía en Iberoamérica no era denominado según su idioma, es decir, rock en español, sino que simplemente se le llamaba rock, de una u otra forma, dando a entender de una forma cortante que el rock era de sello anglosajón y americano. 


\section{México: primer compositor y exportador de música rock en español}

Se puede decir que uno de los primeros países de habla hispana en recibir a este género musical fue México debido a la frontera que tiene entre Ciudad Juárez y El Paso. Gracias a su cercanía con la frontera estadounidense, pudo conocer, adaptar y disfrutar muchas de las cosas que el rock estaba ofreciendo en aquellas épocas. La mayoría de canciones de rock grabadas en español que llegaban a la radio iberoamericana eran procedentes de México, siendo su primer gran exponente Enrique Guzmán (primero con los Teen Pops y luego como solista). Compositores como Cesar Acosta, líder de la banda Los Camisas Negras con éxitos como "Zapatos de gamuza azul", "Ahora o nunca" y una nueva versión de "La bamba". Uno de los primeros grupos, si no el primero, en grabar canciones de rock inéditas fue el grupo Los Locos del Ritmo, el cual se da a conocer gracias a un concurso organizado por Telesistema México, el cual les da la oportunidad de tocar en unos de los programas de televisión de Estados Unidos más importantes para la época llamado Original Amateur, dirigido por Ted Mack, obteniendo el segundo lugar y grabando posteriormente su primer disco de rock en el año 1960. Los intérpretes mexicanos de la época tenían tanto afán y ansiedad por demostrar su competitividad musical hacia los americanos, que cuando una canción de rock americano, sonaba por primera vez en territorio mexicano, no se demoraban más de 24 horas en tener una versión en español para aquellos temas. Es así que temas como "Good holly miss holly" de Little Richard se convertiría en "La Plaga", o "Jailhouse rock" de Elvis Presley se llamaría "El rock de la cárcel", y "Pink shoe laces" de Dodie Stevens sería "Agujetas de color rosa" (Betancourt, 2011, p. 29).

\section{Argentina: precursor de los primeros movimientos juveniles del rock en español}

Este país fue el primero en crear movimientos juveniles en toda lberoamérica. Para fines de los años 50 , había programas de televisión que estaban enfocados a los jóvenes y adolescentes que trabajaban por cantar de la misma forma y las mismas canciones que sus ídolos rockeros ejecutaban. La propuesta argentina se caracterizó por ser muy calmada e inofensiva en cuanto a sus bailes y las manifestaciones que este género producía. De hecho, se apreciaba mucho más la música desde un punto intimista y auditivo, llegando así a tener bailes ordenados, con tintes coreográficos. Uno de los eventos que impactó a la juventud argentina fue la llegada en el año 1955 de la película "Blackboard Jungle" dirigida por Richard Brooks, teniendo como canción principal el tema "Rock around the clock" compuesta por Bill Haley \& His Comets, la cual fue una canción emblemática para Rock and Roll de la época, llegando a ser en Estados Unidos la canción número uno Artistas como Elvis Presley, Patt Boone, Buddy Holly and The Crickets, ya eran programados con total libertad por los jóvenes en la radio argentina. En 1959 se hace el lanzamiento de un programa de televisión llamado Ritmo y Juventud, en el cual, los jóvenes tenían un espacio para cantar en español, las canciones rockeras que tanto les fascinaban en inglés. Más tarde saldría el programa radial El Club del Clan, que catapultó a famosos artistas como Palito Ortega, Violeta Rivas, Lalo Frasen, entre otros (Betancourt, 2011, p. 30).

Se reconoce como a uno de los pioneros más grandes del rock argentino a la banda Mr. Roll y sus Rockeros. La identidad del cantante era un total misterio, ya que no revelaba su identidad (aunque ahora se conoce que el hombre era Eddie Pecheninno, de origen argentino). Esto hizo que alrededor de su identidad corrieran muchos rumores, y que la banda estuviera de boca en boca. Pero lejos de que estos rumores los enterraran, esta banda se lanzó al estrellato haciendo una canción llamada "Celos revueltos al ritmo de rock" en 1956. Esta banda inclusive llego a vender más discos en su país que el mismo Bill Haley \& His Comets. Se le reconoce como una de las primeras bandas en incursionar en la industria musical de su país, con la bandera rockera.

Otro de los grandes músicos rockeros que surgió en aquellas épocas fue Palito Ortega. Es innegable la influencia y el alcance que tuvo este tímido hombre, quien antes de ser músico y rockero se dedicaba a vender café. Este hombre sale a la fama por medio del programa radial El Club del Clan. Algunas de sus canciones más reconocidas son: "Despeinada", "La Felicidad", "Buena gente", éxitos que en su tiempo tuvieron mucha acogida y que hoy en día todavía perduran a pesar del tiempo. Entre otros, que también dejaron huella dentro de esos primeros años de rock argentino se encuentran: Nick Jones, Mary Cosens, Johnny Tudesco 


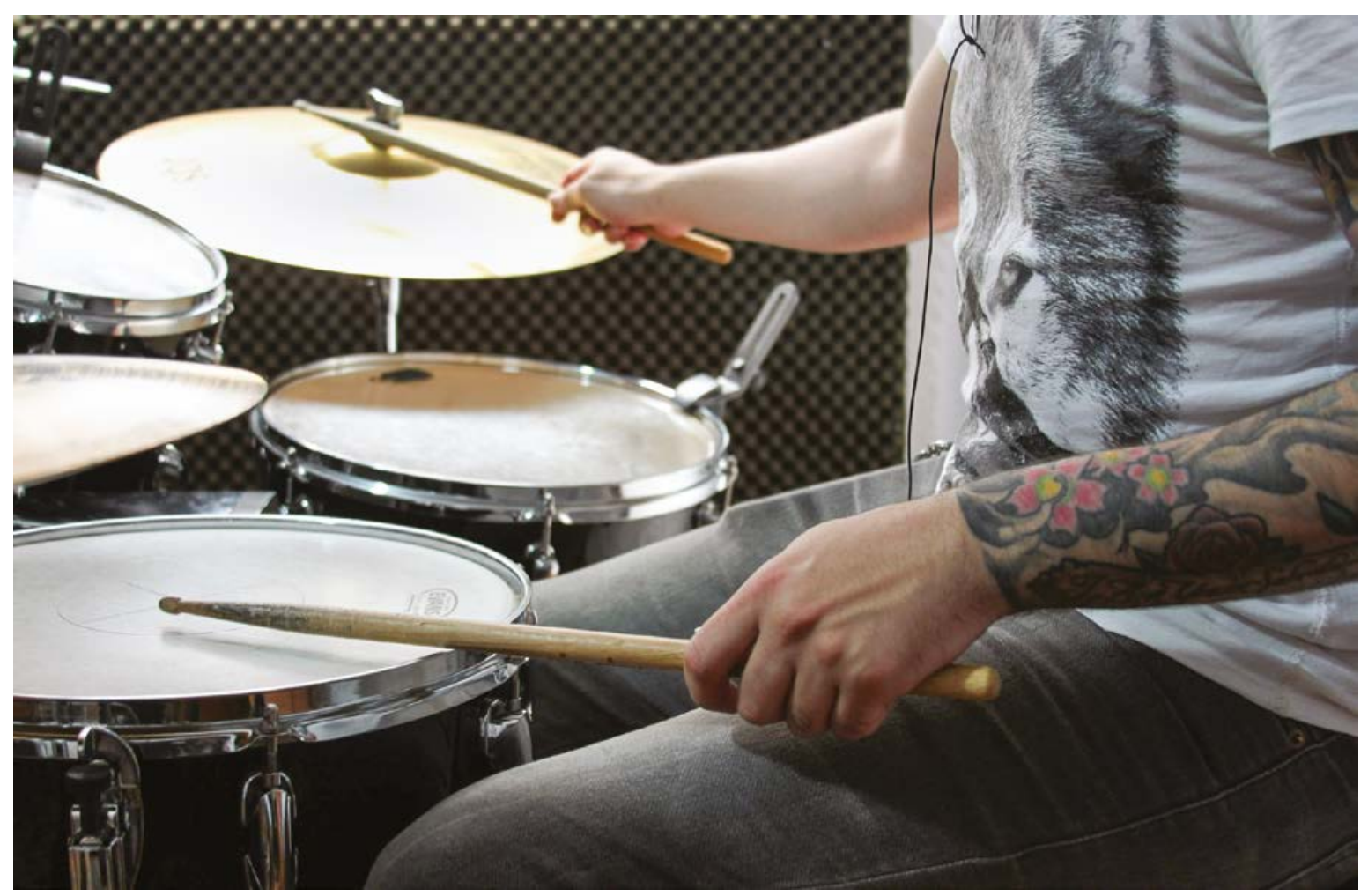

Imagen 1. Camilo Ortegón (2018). Práctica. Fotografía: Pedro Pablo Gómez.

en la parte solista y en los grupos estaría Los 5 Latinos (con su vocalista Violeta Rivas) y los T.N.T (grupo musical italiano radicado en Argentina), (Betancourt, 2011, p. 31).

\section{España: la llegada tardía del rock a la península ibérica}

Debido a la cercanía con Inglaterra, España empieza a crecer y a trabajar en el rock con la misma fuerza como la hacen los ingleses. Pero el avance de este, se vio entorpecido por la dictadura de Francisco Franco, quien se encargó de entorpecer y detener muchos de los avances de este género en el país. Este expresidente y dictador español, pensaba que la juventud debía ser protegida de toda influencia cultural nociva, llamando de esa forma a la música que producía por ese tiempo Elvis Presley, Paul Anka, entre otros. También se encargó de cerrar toda entrada de discos de rock y de detener cualquier clase de manifestación de apoyo hacia ese género en su país, manteniendo la premisa de que el Rock and Roll era una influencia destructiva para la juventud española. Sin embargo, por cosas que pasan en la vida, llega a España la película King Creole protagonizada por Elvis Presley, con su canción "Hard headed woman", como banda sonora de la película. Dos jóvenes llamados Manuel de la Calva Diego y Ramón Arcusa Alcón, a raíz de esta película, empiezan a cantar en pequeñas reuniones, en empresas y locales de la cuidad y es así como se crea el grupo de rock español: El dúo dinámico. (Betancourt, 2011, p. 33).

Uno de los logros más importantes de este grupo fue ser uno de los primeros en Iberoamérica en grabar rock en su idioma con sus propias composiciones. De hecho, este grupo llega a ser muy popular al punto de prensar sencillos (discos de una o dos canciones), de forma continua en su país. Canciones reconocidas como "Quince años tiene mi amor", "Perdóname", "Esos ojos", fueron algunos de sus éxitos. Sin embargo, para el año 1972 se retiran de los escenarios para componerle canciones a Camilo Sesto, Manolo Otero, Julio Iglesias, entre otros reconocidos cantantes de música de plancha. 
En 1958, llega a Madrid el grupo Los Llopis, quienes venían de Cuba y producían un rock con elementos de la música afrocaribeña. Su llegada a España se prestó para que muchas cosas del rock pudieran evolucionar tanto a nivel musical, como a nivel industrial. De hecho, este grupo se encarga de dar a conocer canciones en inglés con su correspondiente traducción en español. Canciones como "See you later aligator" de Bill Haley \& The Comets y su versión en español llamada "Hasta luego cocodrilo", "All shook up" de Elvis Presley en español llamada "Estremécete", "Don't be cruel" de Elvis Presley en español llamada "No seas cruel", son algunas de las canciones que este grupo da a conocer en su idioma. Dos cosas que sucedieron para que esta fuerza imparable del Rock and Roll no se detuviera en España, además de todo lo anterior, fue la llegada de cantantes y bandas provenientes de Argentina tales como Enrique Guzmán, Billy Carafaro y los 5 latinos. Además de esto, llegan los discos originales de los cantantes americanos que estaban en su auge en ese momento. Elvis Presley, Budy Holly, The Crickets, Little Richard, hicieron que los jóvenes empezaran a buscar aun en el sonido de sus guitarras rudimentarias y clásicas, el sonido de un rock que pudiera expresar todo lo que ellos estaban sintiendo y percibiendo en ese momento. Esto ayudó mucho a que los jóvenes ya no estuvieran más reprimidos por la presión del gobierno.

Dos de las ciudades en donde hubo más reconocimiento y avance del rock fueron Barcelona y Madrid. Por Barcelona, el representante fue la banda El dúo dinámico y por Madrid, fue la banda Los estudiantes. Mientras tanto, bandas como Los pájaros locos, Los Mustangs y Los Siries, empezaban a tocar en diferentes partes del país extendiendo la oleada del rock. De hecho, la banda Los Mustangs hace la versión en español más conocida de la reconocida canción de The Beatles "The yellow submarine". Esto produjo que cada vez las industrias discográficas españolas se interesaran más por el producto de rock que estaban generando estos músicos y poco después se empezaron a hacer festivales de rock. Pero por respeto a los valores tradicionales y políticos que regían en ese momento España, en el año 1964, los eventos de rock se cancelan a causa de que se pensaba que la música era provocadora y que las vestimentas de los jóvenes eran grotescas e indebidas (Betancourt, 2011, pp. 34-35).

\section{Brasil: la tierra inconquistable en la que el rock no pudo entrar}

En la lista de los países en los que la ola del rock iba a pegar con fuerza, estaba Brasil. Este país contaba con todos los puntos favorables para que esto sucediera. De hecho, según el censo del 2014, Brasil cuenta con 204.450.649 habitantes, y además de esto es uno de los países con la economía más fuerte en Suramérica. También, este país cuenta con un alto porcentaje de personas de raza afro, lo que sugeriría que aceptarían el rock, por sus raíces con el blues. Sin embargo, uno de los problemas más graves para que el auge del rock pudiera expandirse era el idioma. Cabe aclarar que la lengua nativa de Brasil es el portugués y que el $85 \%$ de las personas de este país hablan este idioma. Esto hizo que las puertas se cerrarán a muchas de las cosas que estaban sucediendo en el mundo por aquella época y que Brasil contemplara lo que estaba pasando dentro de sus territorios. Mientras de los años 1960 a 1970 de desarrollaba en el mundo el auge del rock, tanto en América, Iberoamérica, y otros países de origen anglosajón, en Brasil aparecía uno de los más grandes reconocidos músicos del Bossa Nova llamado Joao Gilberto, incursionando en un género rico en trabajo musical (Betancourt, 2011, p. 35).

A la mitad de la década de 1960-1970, la Bossa Nova pierde fuerza entre los jóvenes brasileños los cuales sienten que este género musical no los identifica y empieza a surgir un nuevo estilo llamado Tropicalismo, en el cual se mezclan las melodías de la Bossa Nova con letras de música protesta. De hecho, se culpa al avance de este estilo como el que condenó a que el rock no tuviera el avance que podría haber tenido en este país. Algunos de los cantantes más reconocidos de este estilo son Gilberto Gil, Caetnao Veloso y Tom Ze. Tristemente el rock no llega a Brasil con la fuerza y la influencia que se esperaba, sino que llega como un producto de consumo más entre la música. Una de las bandas que se aventuró a hacer un esfuerzo grande para que el rock brasilero tuviera un desarrollo, fue una banda llamada Os mutantes, nacida en 1966, fue el primer grupo de rock de este país. Les gustaba hacer rock sicodélico combinando sonoridades de distintos tipos. Tristemente esta banda no tuvo el alcance que se desearía y años más tarde se disuelve. Solo se sabe que la banda volvió a aparecer años más tarde, después de que Kurt Cobain, 
ex cantante del grupo Nirvana, los llamará para que fueran teloneros de un concierto de su banda en Brasil. Se volvieron a reunir para tocar en este concierto, pero ya los músicos estaban enfocados en otras cosas (Betancourt, 2011, p. 36).

\section{Llegada y desarrollo del Rock and Roll en tierras colombianas (1957 - 1965)}

En uno de los documentos más relevantes para la historia del rock en nuestro país, Umberto Pérez muestra la llegada del rock a Colombia narrándolo de la siguiente forma:

De la misma forma que los colombianos se habían enterado de todas las vicisitudes de la Segunda Guerra Mundial, de lo que aconteció inmediatamente después del asesinato del caudillo Jorge Eliecer Gaitán y de las atrocidades cometidas durante el periodo de La Violencia, se enteraron de la existencia de un nuevo ritmo musical que escandalizaba a la sociedad norteamericana: el rock and roll. La radio fue el medio de comunicación por excelencia en Colombia hasta bien entrados los años sesenta. Era a través de ella que los colombianos, tanto en el campo como en la ciudad, se enteraban de lo que acontecía en el mundo entero (Pérez, 2007, p. 27)

De esta forma llega el Rock and Roll a Colombia. La música que se reproducía por la radio nacional era de tipo tropical, clásico, rancheras, boleros, entre otros. Hasta que el rock encontró un espacio para ser reproducido por medio de Jimmy Reisback, ${ }^{1}$ hijo de padre escoces y madre colombiana, en la emisora Nuevo Mundo que era parte de Caracol Radio en la cuidad de Bogotá. Este programa tenía una frania especial, de once a once y media de la noche y allí se reproducian reconocidas canciones de rock por aquellos tiempos. Reisback, recién llegado a Bogotá había estudiado en Estados Unidos, trae consigo discos de los artistas de rock más importantes en aquellos tiempos: Elvis Presley, Budy Holly, Chuk Berry y Little Richard. Este programa en principio empezó en aquel horario para la audiencia de personas adultas. Al principio no pareció gustarle mucho a la audiencia adulta puesto que estaba acostumbrada a escuchar boleros o música tropical, pero a la emisora le pareció

\footnotetext{
1 Jimmy Reisback, llegó a ser años más tarde uno de los mejores abogados del país y a tener en su bufete a 50 abogados (El Tiempo, 2 de julio de 2011)
}

un formato innovador que decidió dejar al aire y que tiempo después se encargaría de tocar a un grupo en especial: los jóvenes (Pérez, 2007).

Además de la aparición del Rock and Roll en la radio colombiana, este género tendrá su aparición en el cine por medio de las películas que contaban con una banda sonora rock. Películas como Semilla de maldad (The blackboard jungle), Rebelde sin causa (Rebeld without a cause) y iSalvaje! (The wild one) fueron tan fuertes y determinantes en la vida de los jóvenes que marcaron aun la forma en la cual se vestían y se peinaban. Además de esto, las canciones que reproducía en la radio Reisback, respaldaban este movimiento que el rock estaba empezando a gestar en medio de los jóvenes de aquella época. Aparecen otros disc-jockeys como Carlos Pinzón, el cual dirigía un programa que salía al aire una vez a la semana en la emisora Nuevo Mundo llamado "Monitor" y a través de este programa, Pinzón empezó a promocionar discos y películas que estaba en ese momento en auge. De hecho, una de las más películas más importantes del momento se llamaba "Al compás del reloj" (Rock around the clock), que se estrenó en el teatro el Cid de la cuidad de Bogotá, teniendo una campaña muy fuerte antes de su estreno, con la canción Rock around the clock del artista Bill Haley and His Conets. El impacto que tuvo esta película fue muy grande en la juventud bogotana, y no solo esta película, sino todas las que vendrían tiempo después con un contenido de rock. De hecho, los jóvenes contaban con concursos de baile, en los cuales debían bailar de la forma más parecida posible a las personas que aparecían en los filmes. Esto hizo que esta película durara varias semanas en cartelera y que el programa tuviera una oportunidad para rifar boletas, hacer concursos de baile y fiestas, aprovechando el gran impacto que tuvo esta película en la juventud bogotana y además que este programa tuviera un seguro posicionamiento a nivel nacional (Pérez, 2007).

A finales de la década de los 50, empiezan a aparecer en Bogotá grupos de rock como los Los Danger Twist y Los Dinámicos los cuales eran de los más reconocidos, Los Strangers, Los Be Boops, Los Silver Thunders, Los Caminantes, Los Desconocidos, Los Rebeldes y Los Electrónicos, eran bandas que se empezaban a consolidar y que para ensayar se reunían en la casa de alguno de 
sus integrantes. Hay varios factores que se deben notar en el surgimiento y levantamiento de estas bandas. El público que tenía el rock era pequeño, puesto que, hasta ahora iba creciendo el impacto de este género sobre la capital. El hecho de obtener instrumentos era algo que muy difícil ya que en el país no se fabricaban instrumentos como guitarras eléctricas, baterías, teclados o bajos eléctricos y además de esto la importación de algún instrumento era muy costosa. Por ende, los músicos se valían de sus conocimientos para construir sus propios instrumentos y así avanzar en los proyectos musicales. Un ejemplo de esto es que, para emular el sonido de una guitarra eléctrica, había personas que extraían un micrófono que se encontraba en los teléfonos públicos y se lo adherían a una guitarra acústica (Pérez, 2007).

Según Umberto Pérez, la primera banda de rock bogotana en grabar un disco de larga duración o un LP, fue la banda Los Daro Boys, con su disco llamando "Los Daro Boys en el Colón". Esta banda estaba conformada por siete estudiantes de la Universidad Nacional, que tocaban con un formato de guitarras acústicas y una batería un poco incipiente como lo narra Pérez, un rock que era mezclado con ingredientes de Bossa Nova y Jazz, de ahí que se les denominara como músicos modernistas y evolucionistas. Pero además de incursionar con estos ritmos, también tocaban música rock mezclándola con sonidos andinos y de música colombiana, como por ejemplo, el Bambuco. Esta mezcla de ritmos le interesó a Simón Daro, propietario de la disquera Discos Daro, quien hizo con la banda un contrato en el cual les ofrecía la grabación de varios discos. De hecho, el nombre de la banda se debe a la cobertura de la casa disquera sobre la banda (Pérez, 2007).

Uno de los derivados del rock que empieza a adquirir fuerza en la música de estas épocas es el llamado Twist. Felix Riaño, reconocido periodista y experto en rock (2006), citado por Pérez en su trabajo, hace este comentario de análisis musical al rock que se tocaba, y además hace una pequeña alusión al derivado que estaba adquiriendo fuerza, el Twist:

El compás de cuatro cuartos no tenía ninguna complicación para la ejecución de las guitarras, el contrabajo o la batería. Esta última sólo marcaba cada tiempo dentro del ritmo. El compás tiene cuatro tiempos, de los cuales dos son fuertes y dos débiles dispuestos intercaladamente; los débiles se marcaban con el bombo y los fuertes con el redoblante [y en el twist] el tiempo fuerte del compás de cuatro cuartos se marcaba con dos golpes de redoblante en la batería (Pérez, 2007, p. 38).

Varios lugares de la capital estaban siendo invadidos por el rock y cada vez la afluencia de gente era mayor. Chapinero y el centro de la cuidad, fueron lugares claves para el desarrollo del rock en Bogotá. El Café Cisne, que estaba ubicado en la capital y el almacén A. Pamp, eran los lugares de reunión y encuentro de los jóvenes de esa época. También en el edificio Colpatria se reunía jóvenes intelectuales y músicos para tertulias a plena luz del día. De hecho, en el almacén A. Pamp, se produjo la primera presentación en vivo de la banda Los Dinámicos y vale recordar que dentro de este grupo se encontraba uno de los músicos que marcaría indiscutiblemente una gran huella en la historia del rock colombiano, él es Humberto Monroy, quien más tarde estaría en bandas como Los Speakers, Siglo Cero y Génesis de Colombia. Cada vez el grupo de jóvenes que escuchaban rock era mucho más grande y las bandas que se formaban cumplían con su deber de ensayar y añorar tener un concierto para el público rockero que iba en crecimiento. Indudablemente una de las cosas que soñaba cada banda en estas épocas era poder tocar al lado de un músico extranjero ya que esto les traería reconocimiento ante la comunidad del rock. Pero uno de los factores que afectó grandemente a las bandas que surgían era que muchas de ellas no contaban con un material grabado y el método de "voz a voz" no era muy efectivo y no propiciaba una ayuda del público para que el trabajo de estas agrupaciones se pudiera consolidar (Pérez, 2007).

\section{Los Speakers: el nacimiento de una banda icónica del rock colombiano}

En el año 1964, Caracol logra traer a Colombia uno de los cantantes más reconocidos del rock en español y que viene a traer la nueva ola mexicana, este hombre es Enrique Guzmán, reconocido por ser uno de los cantantes más prominentes en los Teen Tops. Su carrera en solitario fue exitosa gracias a interpretaciones con Paul Anka y Neil Sedaka, artistas anglosajones. Cuando Guzmán vino a Bogotá, en realidad no hubo necesidad de que se le hiciera mucha publicidad como Caracol 
acostumbraba a hacer con los artistas que manejaba, porque en realidad Guzmán era tan conocido en el rock iberoamericano como el mismo Elvis Presley. Este artista tuvo su presentación en el pabellón de la Feria de Exposición de Bogotá lo cual produjo que los empresarios y productores se reunieran y decidieran crear dos bandas nacionales para que hicieran un contrapeso al artista mexicano, y así nacieron bandas como Los Dinámicos y Los Rebeldes. Sin embargo, un efecto colateral que tuvo la presentación de este mexicano, es que sin pensarlo ni planearlo, nace una de las bandas más icónicas del rock colombiano, Los Speakers. Esto se produjo porque los empresarios decidieron hacer un supergrupo o una super banda uniendo a Los Dinámicos y Los Rebeldes puesto que la calidad musical era muy baja y se necesitaba una buena representación de Colombia esa noche y cada banda tenía elementos que le faltaban a la otra. Además de esto se suma que las bandas tenían un papel de antagonismo entre ellas mismas y esta idea se vino abajo. Pero algún tiempo después, Los Rebeldes invitaron a Brando Ortiz, miembro de Los Danger Twist, y los Dinámicos invitaron a Luis y Edgar Dueñas, integrantes de la banda Los Electrónicos, también hijos del reconocido compositor y maestro de música colombiana Luis Dueñas Perilla y es allí donde nace el reconocido grupo Los Speakers, que se consolidaría con el sonido del guitarrista Rodrigo García. Esta banda era una de las que tenía más nivel musical en aquellos tiempos, ya que muchas de las bandas de rock de aquellas épocas tenían un trabajo empírico en sus instrumentos y no tenían un buen nivel instrumental y como banda. Este grupo estaba conformado del siguiente modo: Humberto Monroy, bajo y voz; Rodrigo García, teclados y voz; Luis Dueñas, guitarra y voz; Oswaldo Hernández, guitarra y voz, y Fernando Latorre, batería. (Pérez, 2007).

Con el nacimiento de Los Speakers y la llegada del rock a la radio, el panorama que tenía el rock por delante era muy alentador. De hecho, la industria discográfica empezó a interesarse por las bandas de rock que estaban empezando a emerger y que tenían una buena calidad musical. Sello Vergara, casa disquera que trabajaba música popular entre ellas rancheras y boleros, se interesó por Los Speakers y graba su primer álbum titulado con el mismo nombre de la banda. De hecho, esta banda tendía a imitar o más bien, a apropiar el estilo "Beatle" que era muy común en la época y que dejaría una importante marca no solo en Los Speakers sino en todas las bandas de rock que estaban surgiendo en ese momento en la capital.

\section{Fernando Latorre, primer baterista de Los Speakers}

Fernando Latorre, baterista que grabó con esta banda los dos primeros discos llamados "The Speakers" (1965) y "La Casa del Sol Naciente" (1966), se retira de la banda después de grabar estos dos discos puesto que estaba estudiando arquitectura en la Universidad de América y se le presenta la oportunidad de viajar al exterior. Latorre, el 9 de mayo del año 2012, le brinda una entrevista a la W Radio en la cual habla un poco acerca de su vida y trayectoria musical y cuenta con que casas disqueras grabó los dos primeros discos de Los Speakers, su viaje al exterior, su estadía en Sudáfrica por 20 años y algunos otros detalles.

\section{Los Flippers: nacen las "aletas" del rock colombiano}

Uno de los lugares reconocidos y frecuentados por la juventud bogotana en los años 60 era una discoteca llamada La Gioconda, la cual estaba ubicada en el Pasaje El Libertador, en Chapinero. Los jóvenes rockeros encontraron un espacio en el cual se podían reunir para escuchar sus bandas favoritas en vivo, la mayoría de rock como en el caso de Los Speakers. Muchos de los jóvenes que asistían a este tipo de reuniones eran menores de edad, por lo tanto, el consumo de alcohol era prohibido y esto hizo que los jóvenes empezaran a consumir Coca-Cola en vez de alcohol. De allí el termino Cocacolos. Ahora los jóvenes estaban más cerca de sus artistas favoritos, al punto que podían ver sus ensayos y hasta entablar con ellos una amistad. Es así como nace la banda Los Pelos, conformada por Jorge Latorre, hermano de Fernando Latorre baterista de Los Speakers, Carlos Piñeros y Miguel Durier. Estos músicos se conocieron durante los ensayos de Los Speakers y después de entablar una amistad empezaron a hacer un proyecto musical. Aunque la banda tuvo oportunidades de ser teloneros de Los Speakers en sus presentaciones, no duró mucho puesto que Durier, un excelente guitarra, pasaría a ser guitarrista de otra de las 


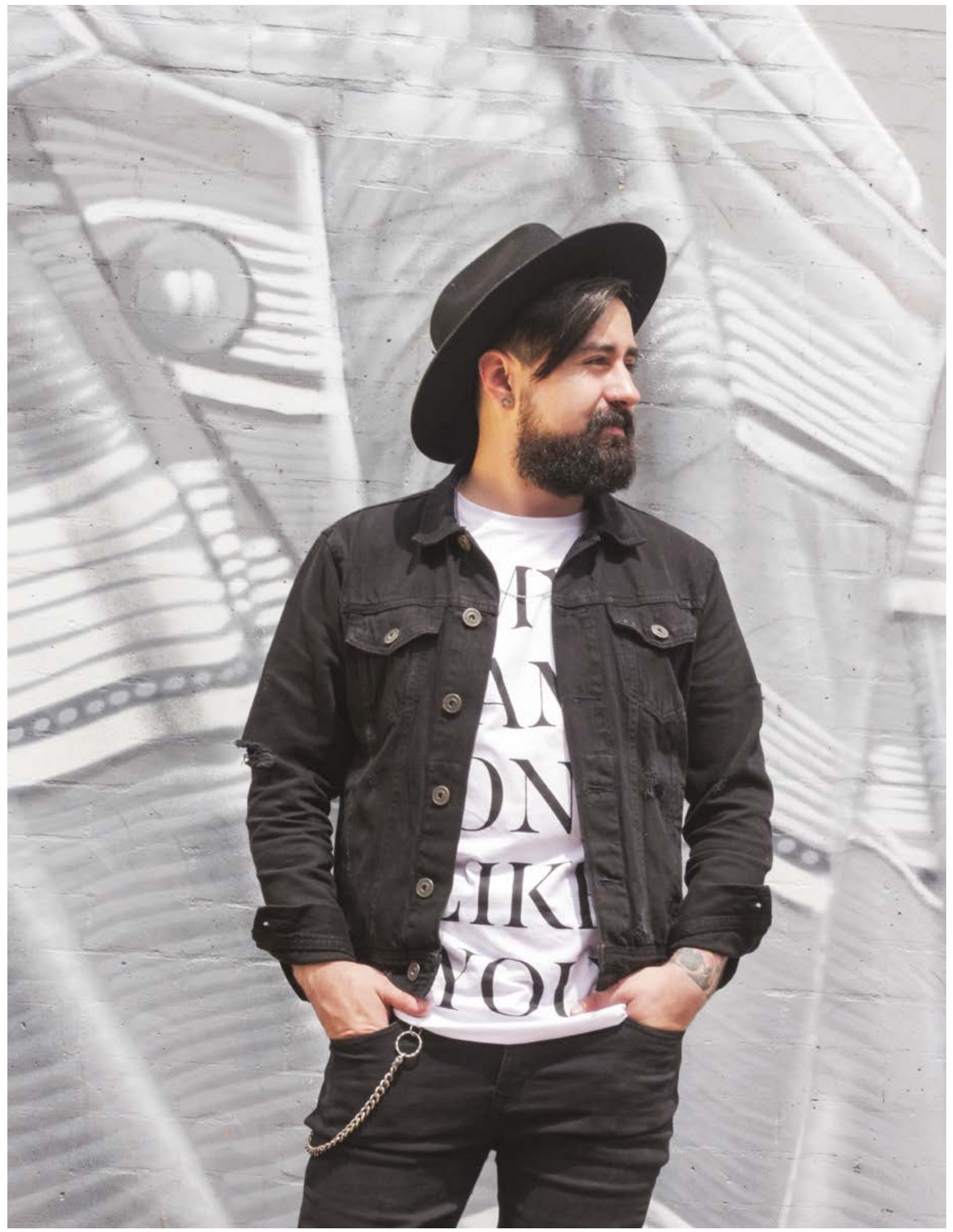

Imagen 2. Camilo Ortegón (2018). Fotografía: Stratósfera Media. 
bandas más reconocidas e importantes del rock colombiano, Los Flippers (Pérez, 2007).

Esta agrupación nace a partir de una banda llamada Los Thunderbirds, compuesta por el ex baterista de Los Speakers, Edgar Dueñas, Carlos Martínez en el bajo, Orlando Betancur en la guitarra y Arturo Astudillo, quien actuaba como guitarrista y director de la banda. Miguel Durier y Guillermo Acevedo, entrarían después de la salida de Dueñas y Betancur. Dueñas entraría de nuevo a las baterías de Los Speakers, después de la salida de Latorre y ahora el nuevo baterista de este grupo sería Guillermo "Memo" Acevedo. Esta agrupación graba en el año 1966 el disco "The Flippers Discotheque" para el sello discográfico Zeida Discos de la cuidad de Medellín. Aquí se puede apreciar también que no solo Bogotá era una ciudad en la cual el rock tenía movimiento, sino que Medellín pasaría a ser una ciudad en la cual el rock encontraría un espacio y un desarrollo. Esta banda tuvo mucho acercamiento con Los Speakers puesto que su manager era el mismo, Edgar Restrepo Caro. Estas dos bandas tuvieron la oportunidad de viajar al exterior para comprar sus propios instrumentos y amplificadores para mejorar su sonido rockero. Los Speakers viajaron a Venezuela y Los Flippers viajaron a Miami (Pérez, 2007).

\section{Guillermo “Memo" Acevedo: el famoso ex baterista de Los Flippers}

Guillermo "Memo" Acevedo, tocaba batería en su casa por simple hobby. De hecho, la experiencia de como él llega a ser baterista es gracias a que cuando tocaba batería, Carlos Martínez y Arturo Astudillo pasaban por su casa admirando su talento, hasta que pasados seis meses le ofrecieron que fuera parte de la banda The Flippers. Después de haber visto una película sobre el legendario baterista americano Gene Krupa, Acevedo decide que su vida sería la música. Empezando su carrera con Los Flippers, tres años después decide dejar la banda para buscar crecer en su instrumento; se une a Yamel Uribe, Ramiro Lozano para experimentar géneros extranjeros. Esto lo llevo a crear el grupo musical La cuarta dimensión. Apasionado por el Jazz y por las músicas extranjeras, viajó a Europa y estudia música con el maestro Enrique Llierte. Después de buscar por un año trabajo, entra al grupo Los Pequeniques, el cual era muy reconocido en España en esos días. Después de dos años se abre paso viajando a México, Canadá y Estados Unidos. En el año 1977 se radica en Canadá y crea el grupo de salsa llamado Banda Brava, siendo uno de los primeros (sino el primero) grupos de salsa en este país. Allá estuvo nominado a un importante premio musical que es equivalente a los Grammy, en la categoría World Beat. Después de 13 años conforma un grupo de Latin Jazz llamado El Cartel del Jazz, que al principio fue criticado por su nombre, pero aceptado por su música. De hecho, Acevedo mismo cuenta que al mes de haber salido el primer disco de este proyecto, en Estados Unidos y Canadá, ya llevaban vendidos alrededor de 1.700 copias. Hoy en día está radicado en New York, dedicado a la docencia y a la difusión del sonido latino (Eje 21, citado en Revista Semana).

En una entrevista hecha por la revista online Eje 21, en octubre de 2014, Acevedo habla un poco acerca de su vocación música, su primera agrupación, su trabajo con los Flippers, entre otras experiencias de su vida.

\section{La influencia de la estación Radio 15}

Radio 15 era una estación de radio que hacía parte del grupo Caracol, tenía en aquellos tiempos un programa llamado "El show de los frenéticos" dirigido por Carlos Pinzón y Alfonso Lizarazo. Este programa tuvo una gran influencia en la juventud de aquellas épocas y también se encargó de apoyar las bandas de rock nacional y cantantes solistas que en aquel momento empezaban a nacer. Fue clave en el apoyo a Los Speakers como banda, y también a un grupo musical nuevo que se llamaría Los Ámpex. Este grupo se deriva de una banda llamada los Goldfinders que a su vez también estaba conformada con músicos que habían salido del grupo Los Rebeldes. Los Ámpex, estaba conformada por el caleño Óscar Lasprilla en la guitarra y la voz, Yamel Uribe en el bajo, Jaime Rodríguez en la guitarra y la voz, y Óscar Ceballos en la batería. En su repertorio se podían apreciar canciones de grupos americanos y anglosajones, pero con un sonido mucho más pesado, como el sonido de la banda The Rolling Stones, que el público no aceptaba del todo. Aunque bien, la banda era aceptada entre la gente por causa de su naturalidad. Los cantantes también empezarían a tener una aceptación entre el público y un apoyo notable por parte de Radio 15. Cantantes oriundos como Harold Orozco que se hizo famoso por la canción "Michey Mouse" 
y Óscar Golden con canciones como "Boca de chicle" y "Zapaticos pom pom". Estos dos cantantes marcarían el inicio de una nueva ola musical a finales de la década de 1960 (Pérez, 2007).

Aunque Radio 15 era una emisora joven que tenía programas innovadores y frescos para aquella época, no contaba con el cien por ciento de apoyo de parte de sus directivas, puesto que se decía que era una estación para los jóvenes. La televisión también estaba dando sus primeros pasos y la cantidad de horas diarias que se transmitían eran cuatro, nada más. Entonces los jóvenes no tenían otro plan que los pudiera distraer que escuchar esta emisora que transmitía música rock tanto nacional como anglosajona y americana. La reacción en los jóvenes con respecto a la música rock ya no era tan pasiva como en los primeros años y se empezaba a sentir dentro de los jóvenes un aire de inconformismo con la sociedad ordenada y tradicionalista en la que habitaban. Es entonces cuando el aspecto físico de los jóvenes empieza a cambiar: el cabello largo, ropa de colores, el querer dedicar su vida a la música rock; las mujeres vestían faldas cortas, eran algo extrovertidas y ahora salían sin permiso de sus casas saliendo con sus pretendientes. Estos comportamientos de aquellos jóvenes eran solo una forma de protesta a una sociedad tradicionalista y también una señal de inconformidad con muchos de los sistemas culturales y políticos de aquel entonces (Pérez, 2007).

\section{El plano musical rockero desde 1966 a 1970: Los Flippers y Los Speakers}

El crecimiento del rock en estos años fue algo grande y muy bueno. En estos años la actividad musical rockera se consolidó mucho más y ahora las bandas estaban mucho más estables en cuanto a su formación. Aunque bien salían algunos músicos de las bandas conformadas, los miembros fundadores seguían. Además de esto, las casas disqueras empezaron a darle la oportunidad a muchas más bandas que grabaran sus discos de rock, algo que en años anteriores no se veía o se consideraría muy difícil. Ahora había más bares, más discotecas, y las bandas de rock empezaron a ser las bandas locales de estos lugares y esto hacía que las bandas, ahora al tener mucho más acercamiento con sus instrumentos, tuvieran un desarrollo más profesional y en proceso de creación más profundo. Una de las ventajas de estos lugares en los que las bandas reconocidas de rock de aquellos tiempos pudieran tocar en vivo sus canciones, era que ahora el rock no era un género de "uso exclusivo" para los jóvenes, sino que ahora personas adultas y hasta personas oriundas de la capital o provincianos, tuvieran la oportunidad de escuchar rock (Pérez, 2007).

Por estas épocas, Los Flippers grabaron su segundo disco llamado "Psicodelicias" con el nuevo cantante y guitarrista Ferdie Fernández, quien se encargó de reemplazar a Miguel Durier que dejó el grupo para hacer parte de 4 Crickets, una reconocida banda de rock mexicana por aquellos tiempos. De hecho, con el título de este disco, ya se estaba empezando a ver cómo sería la evolución del rock en algunos años. También Los Ámpex graban dos discos titulados "Infierno a Go Gó" y el segundo disco con el nombre de la banda "The Ámpex". En 1966 nace una banda llamada Young Beats los cuales aparecen el programa "El show de los frenéticos" de Radio 15, dirigido por Alfonso Lizarazo. En su primera presentación tuvieron muy buena aceptación de parte de las personas y muy buenos comentarios de músicos de otras bandas de rock. De hecho, esto produjo que consiguieran un contrato para grabar su primer disco llamado "Ellos están cambiando los tiempos", el cual ya no tendría un sonido tan beatle, sino un sonido más sucio y más dado al blues, mucho más cercano hacia lo que hacian The Rolling Stones (Pérez, 2007).

En estos años también aparecen importantes bandas de rock como Los Streaks, grabando dos discos y Los Yetis de Medellín con otras dos grabaciones que llevan el nombre de la banda. Aunque vale aclarar que el disco que tuvo mayor impacto en estos años fue "La Casa del Sol Naciente" de Los Speakers, del cual se logró vender unas 15.000 copias, tamaño que era inmenso para la época, aunque ahora mismo en estos tiempos continúa siendo un número de copias bastante amplio. Esto les otorgó un Disco de Oro por ventas. Ahora, había muchas bandas de rock que se empezaban a formar y aunque no todas grababan discos, eran conocidas por tocar en bares reconocidos. Bandas como Los Beatnicks, Los 2+2 (integrados por Augusto Martelo, Christian Gómez, Nano Pombo, Willy Vergara y Diego Betancur, hijo del futuro presidente de la República) y Los Playboys, conformados por Chucho Merchán, Juango Fernández y Eduardo Sardino Acevedo, 
futuros integrantes de importantes bandas bogotanas de finales de los sesenta (Pérez, 2007).

Las discotecas de aquellos tiempos demostraron que el rock tenía mucha acogida en el público juvenil. Por esto los publicistas de la firma Cicolac que representaban en Colombia a la firma Nestlé y su producto Milo, crean un evento de rock con el fin de promocionar este producto delegando como organizador a Alfonso Lizarazo. Este evento se llamaría "Gira Milo a gogó" y estaría en varias de las ciudades más importantes del país. El concierto alcanzó tal aceptación entre las personas que, cada evento estaba a lleno total y la gente recibía con gran aceptación a artistas como Los Speakers, Los Ámpex, Oscar Golden, Harold y al ballet de Katy Chamorro (Pérez, 2007).

Esta serie de conciertos por todo el país dejaría una serie de anécdotas y eventos para recordar. Uno de ellos sucedió en Bogotá, en la plaza de toros La Santamaría, siendo este el primero concierto de la gira. Las personas para entrar al concierto lo único que debían presentar era una etiqueta de la bebida Milo. Pero la lluvia impidió por un largo rato el inicio del concierto, por ende, los equipos de amplificación y el sonido se cubrieron con plásticos para evitar cualquier daño y además de esto ningún músico podía tocar por temor a que fuera electrocutado. Después de que paso la lluvia se esperaba que el concierto comenzara, pero hubo un daño eléctrico en la plaza y se tuvo que cancelar. Esto enfureció al público asistente, el cual salió a la carrera séptima a causar daños y estragos, e incluso algunas personas se atrevieron a asaltar un camión de gaseosas. Entre estos incidentes muchas personas fueron arrestadas y entre los arrestados se encontraban integrantes de la banda Los Flippers, quienes más tarde fueron liberados después de que Edgar Restrepo, su manager, los fuera a recoger. Cuando la gira se trasladó a Medellín, se cuenta que Los Speakers no pudieron entrar al lugar por la cantidad de fanáticos que se les abalanzaban, tanto así que,

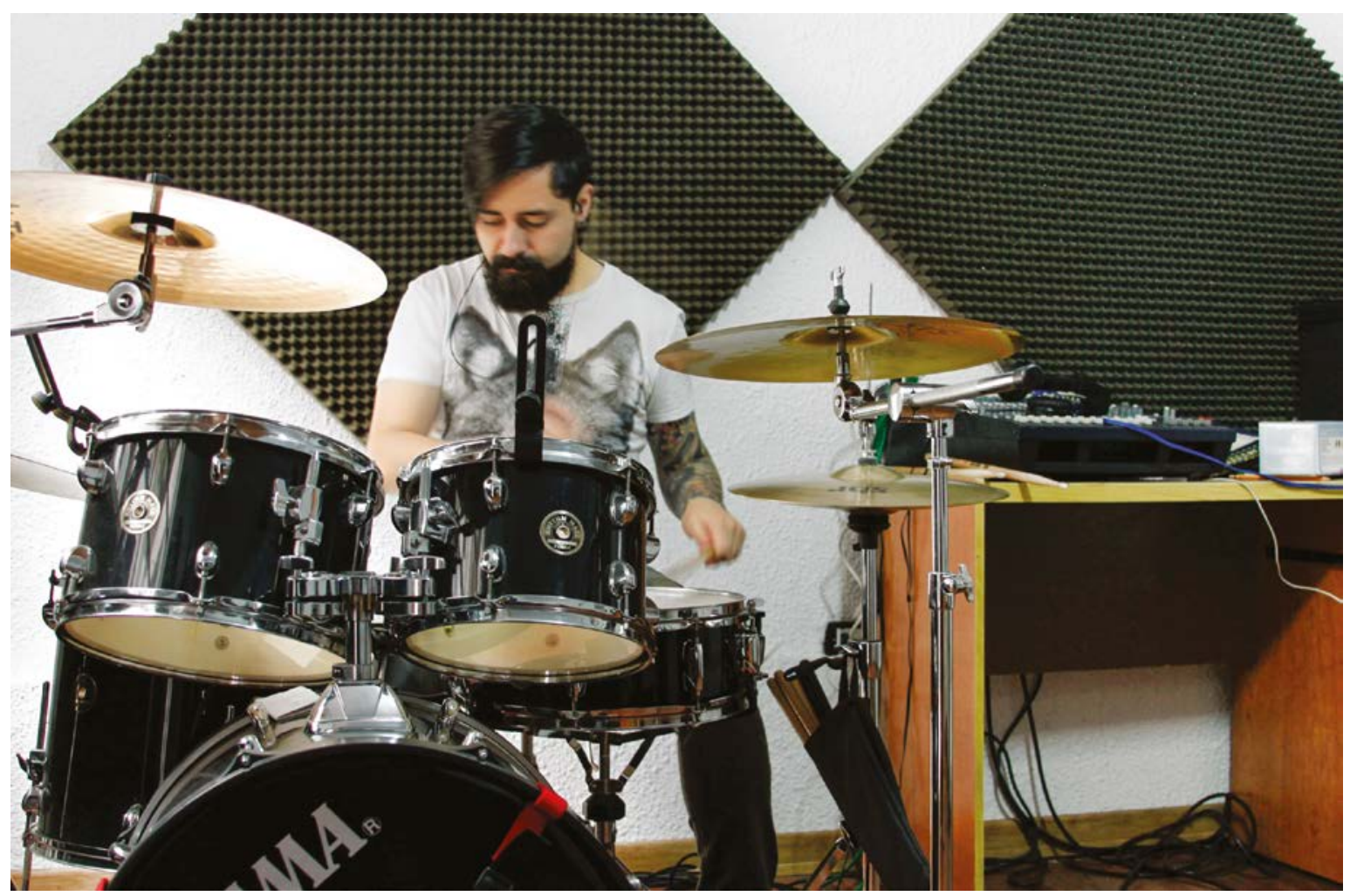

Imagen 3. Camilo Ortegón (2018). Práctica. Fotografía: Pedro Pablo Gómez. 
por querer tocar a los músicos, sus seguidores rompieron los vidrios del carro. Esto hizo que inmediatamente se colocara bajo seguridad a todos los músicos y se cancelara el concierto. En respuesta las personas apedrearon el Palacio del Obispo y la Gobernación, argumentando que la cancelación del concierto había sido por causa de la iglesia católica. Estos estragos causaron que el siguiente concierto que se realizaría en Manizales se cancelara por orden de la Arquidiócesis de allí, argumentando que el rock era una mala influencia para la juventud y promovía comportamientos violentos. Esta gira deja como resultado a finales de 1967, alrededor de 70 conciertos por todo el país, varias anécdotas y recuerdos, y el hecho de que el rock estaba llegando no sólo a los jóvenes, sino a gente adulta, curiosos, niños, entre otros (Pérez, 2007).

En la transición de los años 1967 al 1968, el rock como se conocía empezó a cambiar y a volverse mucho más suave en su musicalidad y en sus letras ya que estaba directamente influenciada por la música anglo de aquellos tiempos y es así como nace una nueva ola. Los ritmos rápidos ya pasaban a un lado y las letras con tinte violento y revolucionario en contra del sistema eran reemplazadas por letras mucho más románticas y suaves. Se podría decir que el rock que antes disgustaba a los adultos, ahora se quería acomodar al gusto de ellos. Vale recordar que lo que pasaba en el exterior con el rock tenía una fuerte influencia en el estilo musical de las bandas y en la ideología de los jóvenes del país. Entonces las ideologías que estaban alrededor del rock a finales de 1967 y 1968 se basaban en creación y pensamiento, un poco más pacifistas, tendrían también su acogida en la juventud seguidora del rock en el país. El hecho de que los ritmos ya no eran tan bailables y pasaron a ser mucho más melancólicos, las letras fueran más reflexivas, la postura del rock ahora dejaba la moda y se volvía un estilo de vida para aquellos que lo querían vivir, produjo que los años siguientes fueran un poco sombríos puesto que a las disqueras lo que les interesaba era vender productos que estuvieran en auge y los siguientes años para el rock no serían muy benignos en cuanto a popularidad, haciendo que el sacrificio de muchos músicos pioneros del rock se olvidara y fuera un poco olvidado. Es así como a finales de la década del sesenta, el rock deja de ser moda a pasar de ser un estilo de vida adoptado por las personas que lo quisieran adoptar (Pérez, 2007).
Al terminar la década de los sesentas bandas como Los Speakers y Los Flippers empiezan a disolverse, pero no de una forma definitiva. También la banda Los Young Beats, quienes conservaban todavía el sonido del rock clásico se disuelve por razones personales de algunos de los miembros y por el panorama que vivía por estos años la música rock en el país. Sin embargo, el final de esta banda produjo como resultado el inicio de otra llamada The Time Machine la cual reunió a Roberto Fiorilli y Fernando Córdoba, ex integrantes de Los Young Beats, con Yamel Uribe y Óscar Lasprilla ex miembros de Los Ámpex. Esta banda mostró al público artistas que hasta el momento no eran conocidos; artistas como Jimmy Hendrix, The Who, Cream y otros. El riesgo que tomó esta banda de presentar otro lado del rock que hasta ese entonces no se conocía, fue significativo pero muy bien recibido por el público seguidor del rock. De hecho, varios músicos de otras bandas resaltaban con muy buenos comentarios el trabajo que hacía la banda al ejecutar canciones de estos nuevos artistas. El trabajo de esta banda fue muy interesante puesto que las canciones que ejecutaban no eran para el público acostumbrado al sonido de la nueva ola, sino para personas que eran aficionadas y seguidoras del rock. Tristemente, por falta de apoyo de los sellos discográficos y de las personas que preferían la nueva ola, la banda se desintegra, aunque dejando una inmensa huella en las bandas bogotanas que más tarde se formarían (Pérez, 2007).

Después de que esta banda desaparece Los Flippers y Los Speakers empiezan otra vez a reaparecer y a reformarse con nuevos integrantes. Es así como Los Speakers grabarían su cuarto disco con Rodrigo García, Humberto Monroy, Roberto Fiorilli y Óscar Lasprilla ex integrantes de The Time Machine. Aunque este disco los hizo viajar a Ecuador, no tuvo la fuerza que se esperaba en los seguidores de la banda. Después de esto Óscar Lasprilla decide dejar la banda para irse a Europa y los tres músicos restantes de la banda deciden sacar un cuarto disco con un concepto muy basado en Sgt. Pepper de los Beatles, pero la casa disquera no los apoyó puesto que consideraba que era un proyecto bastante ambicioso, caro y además, se sumaba que el anterior disco no tuvo los resultados que se esperaban. Después de una larga búsqueda de apoyo aparece Manuel Drezner propietario de los Estudios Ingesón, quien les ayudaría a sacar este proyecto 
adelante dándoles sus estudios para que pudieran grabar. El trabajo que tuvo este disco desde el diseño de la portada, las letras de las canciones las cuales tenían un tinte revolucionario y crudo, ritmos experimentales y psicodélicos, sin nombrar todos los detalles detrás de su creación y su posterior comercialización, se considera uno de los más grandes discos que se hizo en aquellos tiempos. The Speakers en el maravilloso mundo de Ingesón fue el título del disco, el cual contaba con doce canciones con títulos como: "Oda a la muerte mediocre", "Si la guerra es buen negocio, invierte a tus hijos", "Salmo siglo XX, era de la destrucción", significaban una directa critica a la sociedad bogotana y a los atroces eventos protagonizados por Estados Unidos en la guerra de Vietnam. Tristemente el disco no alcanza a tener el impacto que se esperaba y de 1.000 primeras copias que se imprimieron se vendieron 800 . Esto hizo que Rodrigo García tomara la decisión de viajar a España y la banda se desintegra de forma definitiva (Pérez, 2007).

\section{A manera de cierre}

Después de mostrar el plano musical rockero que se veía en México, Argentina, España, Brasil, y por supuesto, mostrar el avance de este género musical en nuestro país; se puede decir que hubo muchos "hilos" a nivel cultural, político, social, religioso, económico que se entretejieron para el avance de este importante género musical, aunque bien es extranjero, tuvo tal capacidad de desarrollarse de una manera imponente y rebelde en el avance cultural en la Bogotá de los años 60, frente a la inconformidad de varia gente de aquella época. Vale aclarar que, aunque la capital no fue la única cuidad en la cual este género musical tuvo grandes avances fue la primera en la cual el rock se empezó a desarrollar y más tarde empezaría a impactar a otras ciudades importantes.

Bogotá, como epicentro del rock entre 1957 a 1975 , fue el elemento de interacción y de reunión de los jóvenes que buscaban disfrutar de este género musical. El rock colombiano logró ser una herramienta que serviría para que los jóvenes expresaran y mostrarán sus preguntas, inquietudes, dudas e inconformidades hacia la sociedad que los rodeaba. Varias de las letras de grupos colombianos rockeros que se escuchaban hacia principios y mediados de los años 60 , tenían un contenido crítico hacia el gobierno y su forma de actuar en el país, e inclusive la música tenía un carácter agresivo hasta que a finales de la década de la década de los 60 las letras románticas y psicodélicas tuvieran su aparición, en donde la música sería un poco más romántica o experimental. También, aunque muchos músicos empezaron imitando a grupos como The Beatles, The Rolling Stone, entre otros, a medida que se desarrollaban también trabajaban con ritmos musicales del país, y así los empezaron a incorporar en sus composiciones. El rock colombiano fue también una clase de manifestación nacional en la medida en que muchos jóvenes en algún momento se sintieron llamados a formar parte de un movimiento con el que se identificaron plenamente; festivales de amplia convocatoria, como los realizados en el Parque Nacional, demuestran que gran parte de la juventud colombiana encontraba en el rock su forma de vida y lo hacían a través de la ideología del hipismo.

Después de haber mostrado una breve historia del rock en Colombia en la década de los 60 , teniendo en cuenta los desarrollos del rock en el contexto mexicano, argentino y brasileño en este mismo periodo, en la segunda parte de este trabajo nos ocuperemos de esta historia entre los años 70 y 90 , ahora intentando visibilizar el papel de los bateristas en el transcurrir de estas historias locales de la música.

\section{Referencias}

Bellón, M. (2009). EL ABC del rock, todo lo que hay que saber. Bogotá, Colombia: Editorial Aguilar, Altea, Taurus, Alfaguara, S.A.

Betancourt, F. (2011). Historia de la música postmoderna en Colombia (Propuesta de documental). Bogotá, Colombia: Pontificia Universidad Javeriana.

Pérez, U. (2007). Bogotá, epicentro del rock colombiano entre 1957 y 1975. Bogotá, Colombia: Secretaria Distrital de Cultura, Recreación y Deporte-Observatorio de Culturas.

Sánchez, G. L., \& García, B. E. (2018). Simbiosis: Composición para bandola andina colombiana y entidades sónicas. Estudios Artísticos, 4(4), pp. 88-109. doi: https://doi. org/10.14483/25009311.12935 